\title{
$\lambda$-Phage Docking Observed at the Single-Particle Level
}

\author{
Ph. A. Gurnev and S. M. Bezrukov \\ Eunice Kennedy Shriver National Institute of Child Health and Human Development, Bethesda, MD, USA; \\ e-mail: pgurnev@helix.nih.gov
}

DOI: $10.1134 / \mathrm{S} 1990747809030246$

We report direct real-time detection of phage lambda docking to its bacterial receptor Maltoporin, a sugar-specific porin of Escherichia coli and Shigella sonnei, in a planar lipid bilayer. Phage binding affects both ion permeation and sugar molecules' dynamics in a single Maltoporin channel. Added from the cis-side, trimeric Maltoporin directionally inserts into a lipid bilayer. In the absence of substrate, the channel is predominantly open, but becomes transiently blocked when maltohexaose is added to the cis-, trans-, or both sides of the bilayer. Wild type phage lambda, added to the cis-side of the bilayer, spontaneously and irreversibly binds to the channel, reducing its ion conductance and completely blocking the cis-side sugar entryway of all three receptor monomers. From the trans-side, sugar molecules still have access to the sugar-binding regions of the Maltoporin pores, however, the residence time of maltohexaose is about twice of that in the phage-free channel. The mean time between blockages of a single Maltoporin monomer (with trans-side maltohexaose application) is independent of the presence of the phage. This finding suggests that phage docking does not significantly change the structure of Maltoporin, and that the phage-binding regions are close to, but do not overlap with, the sugar-binding domains of the receptor monomers. Our analysis shows, however, that ion fluxes through the pores of Maltoporin share a new common pathway in the phage-receptor complex. 